\title{
High Quality Entangled Photon Pair Generation in Periodically Poled Thin-Film Lithium Niobate Waveguides
}

\author{
Jie Zhao® ${ }^{1}$ Chaoxuan Ma, ${ }^{1}$ Michael Rüsing $\odot,{ }^{1,2}$ and Shayan Mookherjea $\odot^{1, *}$ \\ ${ }^{1}$ Department of Electrical and Computer Engineering, University of California, \\ San Diego, La Jolla, California 92093-0407, USA \\ ${ }^{2}$ TU Dresden, Institut für Angewandte Physik, Nöthnitzer Straße 61, 01187 Dresden, Germany
}

(Received 29 January 2020; accepted 7 April 2020; published 24 April 2020)

\begin{abstract}
A thin-film periodically poled lithium niobate waveguide was designed and fabricated which generates entangled photon pairs at telecommunications wavelengths with high coincidences-to-accidentals counts ratio CAR $>67000$, two-photon interference visibility $V>99 \%$, and heralded single-photon autocorrelation $g_{H}^{(2)}(0)<0.025$. Nondestructive in situ diagnostics were used to determine the poling quality in 3D. Megahertz rates of photon pairs were generated by less than a milliwatt of pump power, simplifying the pump requirements and dissipation compared to traditional spontaneous parametric down-conversion lithium niobate devices.
\end{abstract}

DOI: 10.1103/PhysRevLett.124.163603

Spontaneous parametric down-conversion (SPDC) was used to generate the entangled photons used in first demonstrations of quantum teleportation, entanglementbased cryptography, tests of Bell's inequality, one-way optical quantum computing, and other notable experiments [1-8]. Nanoscale waveguides, which reduce the modal cross-section area by 10 times compared to traditional lithium niobate (LN) waveguides, could result in lowering the power requirements by a factor of 100 times and simultaneously improve the quality of the generated photon pairs, by reducing the likelihood of thermal effects or noise contributions from high levels of pump power. This double advantage could enable large-scale multiplexing and scale up toward realistic quantum information processing photonic circuits, which so far is based on other materials with a weaker, third-order nonlinearity like silica or silicon [9-11]. The second-order nonlinear optical process of SPDC in traditional LN waveguides can yield photon pairs of high quality but is relatively inefficient, typically requiring a dedicated tens-of-milliwatt-class single-mode laser diode or a high-peak power mode-locked laser $[12,13]$. Efficient telecommunications-band entangled photon pair generation at submilliwatt power levels has, for example, been developed in silicon photonics, but at the cost of using a weaker, third-order optical nonlinearity and achieving the best performance from microresonator devices, which require stringent control and stabilization

Published by the American Physical Society under the terms of the Creative Commons Attribution 4.0 International license. Further distribution of this work must maintain attribution to the author(s) and the published article's title, journal citation, and DOI.
$[14,15]$. Thus, the realization of high quality entangled photon generation in single pass nanoscale $\mathrm{LN}$ waveguides is under active study (see Table I).

Figure 1(a) shows one of several waveguides fabricated in a $300 \mathrm{~nm}$ thickness $5 \mathrm{~mol} \% \mathrm{MgO}$-doped $x$-cut $\mathrm{LN}$ thin film, with a $1.8 \mu \mathrm{m}$ layer of $\mathrm{SiO}_{2}$ and a Si handle $(0.4 \mathrm{~mm}$ thickness), and using a ridge etch depth of $50 \mathrm{~nm}$. The waveguides have a length of $0.7 \mathrm{~cm}$, with a poled section of length $0.5 \mathrm{~cm}$. The cross section of the selected waveguide is schematically shown in Fig. 1(c). The quasi-TE-polarized fundamental modes, shown in Figs. 1(d) and 1(e), were calculated using vectorial mode simulation software to have modal effective area $A_{\text {eff }}=1.1 \mu \mathrm{m}^{2}$ at $1570 \mathrm{~nm}$ wavelength, and $A_{\text {eff }}=0.4 \mu \mathrm{m}^{2}$ at $785 \mathrm{~nm}$ wavelength. The normalized mode-overlap integral was calculated to be $78 \%$, which leads to a high efficiency of generating photon pairs. However, nanoscale waveguides with small mode cross-sectional area suffer from a large index dispersion, and require a poling period that is about one order of magnitude smaller than in traditional periodically poled lithium niobate (PPLN) waveguides [12,13]. Figure 1(f) shows the effective refractive indices of the modes. Type- 0 quasi-phase-matching (QPM) is used with TE-polarized waveguide modes, which uses the strongest nonlinear coefficient in $x$-cut LN [22]. A first-order grating, which is the most efficient QPM configuration, was achieved by poling the thin film with calculated QPM period, $\Lambda=2.8 \mu \mathrm{m}$, to match the refractive indices of the lowest-order TE-polarized waveguide modes at 1570 and $785 \mathrm{~nm}$. In this configuration, the nonlinear coefficient is $d_{33}=27 \mathrm{pm} \mathrm{V}^{-1}$ at $785 \mathrm{~nm}$.

For $x$-cut thin-film LN (TFLN), surface electrodes are used for poling. During poling, the inverted domains spread quasilaterally from the positive electrodes toward the 
TABLE I. Recent results of entangled photon pair and heralded single-photon generation near $1.55 \mu \mathrm{m}$ wavelengths using optically pumped SPDC in thin-film lithium niobate photonic devices. The quality is defined by the pair flux or pair coincidence rate (PCR), coincidences-to-accidentals ratio (CAR), heralded second-order autocorrelation at zero-time difference $\left[g_{H}^{(2)}(0)\right]$ for a heralded single photon, and the two-photon interference visibility of the photon pair $[\mathrm{V}]$. Values indicated in bold font are record values reported for thin-film LN SPDC devices.

\begin{tabular}{|c|c|c|c|c|c|}
\hline Reference & Structure & PCR & CAR & $g_{H}^{(2)}(0)$ & Visibility \\
\hline Main et al. [16] & Waveguide & [Theory] & $\ldots$ & $\ldots$ & . \\
\hline Frank et al. [17] & Microdisk & $450 \mathrm{kHz}$ & $6^{\mathrm{a}}$ & $\ldots$ & . \\
\hline Luo et al. [18] & Microdisk & $0.5 \mathrm{~Hz}^{\mathrm{b}}$ & 43 & $\ldots$ & .. \\
\hline Rao et al. [19] & Waveguide & $7 \mathrm{kHz}^{\mathrm{c}}$ & 15 & $\ldots$ & $\ldots$ \\
\hline Rao et al. [19] & Waveguide & $28 \mathrm{kHz}^{\mathrm{c}}$ & 6 & $\ldots$ & $\ldots$ \\
\hline Chen et al. [20] & Waveguide & $0.8 \mathrm{MHz}^{\mathrm{d}}$ & $631 \pm 210$ & $\ldots$ & $\ldots$ \\
\hline Chen et al. [20] & Waveguide & $7.2 \mathrm{MHz}^{\mathrm{e}}$ & $23^{\mathrm{f}}$ & $\ldots$ & $\ldots$ \\
\hline Elkus et al. [21] & Waveguide & $36 \mathrm{kHz}^{\mathrm{g}}$ & $6900 \pm 200$ & $\ldots$ & \\
\hline This work & Waveguide & $76 \mathrm{kHz}$ & $67224 \pm 714$ & $\cdots$ & $\cdots$ \\
\hline This work & Waveguide & $672 \mathrm{kHz}^{\mathrm{h}}$ & $8361^{\mathrm{i}}$ & $0.022 \pm 0.004$ & \\
\hline This work & Waveguide & $1 \mathrm{MHz}^{\mathrm{h}}$ & $5807^{\mathrm{i}}$ & $\ldots$ & $\{99.3 \pm 1.9 \%$ \\
\hline This work & Waveguide & $4.2 \mathrm{MHz}^{\mathrm{h}}$ & $1576^{\mathrm{i}}$ & $0.183 \pm 0.03$ & \\
\hline This work & Waveguide & $11.4 \mathrm{MHz}$ & $668 \pm 1.7$ & $\ldots$ & $\cdots$ \\
\hline
\end{tabular}

${ }^{a}$ Estimated from the peak-to-side-lobe $( \pm 0.5 \mathrm{~ns})$ ratio of coincidence counts.

${ }^{b}$ Peak value of the raw coincidence counts divided by the measurement time, and further divided by the detection efficiency and detector gating duty cycle.

${ }^{\mathrm{c}}$ From the stated pump power and on-chip pair generation rate.

${ }^{\mathrm{d}}$ From the stated pair generation rate.

e,f Estimated from Figs. 5(a) and 5(b) in Ref. [20].

${ }^{\mathrm{g}}$ Estimated from the product of the stated values of the loss-corrected normalized brightness, bandwidth, waveguide length, and the losscorrected pump power.

${ }^{\mathrm{h}}$ Estimated from the measured singles rate.

${ }^{\mathrm{i}}$ From the fitted line in Fig. 4(b) at the estimated PCR.

(a)

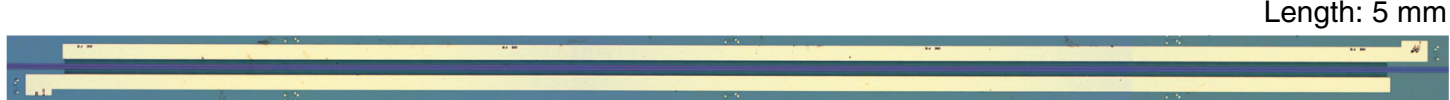

(b)

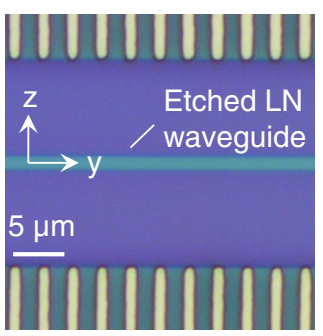

(c)

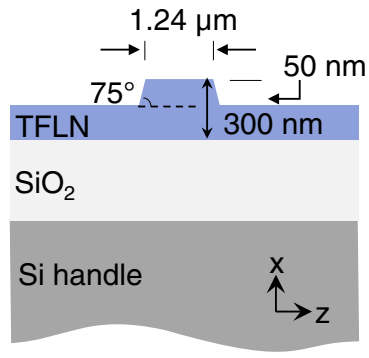

(d)

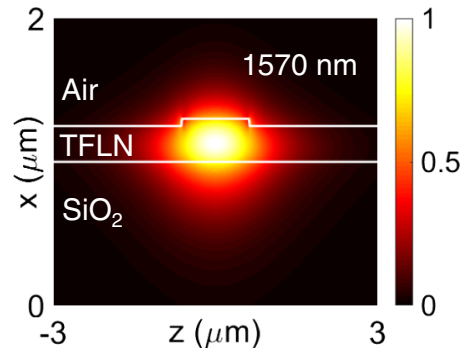

(e)

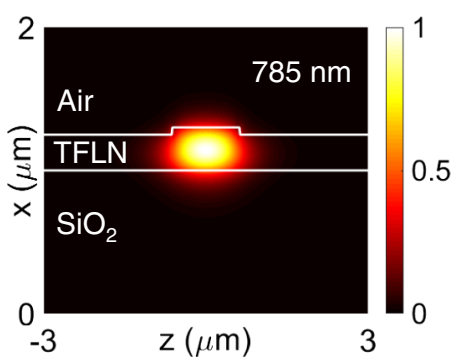

(f)

Effective refractive index $\left(\mathrm{n}_{\text {eff }}\right)$

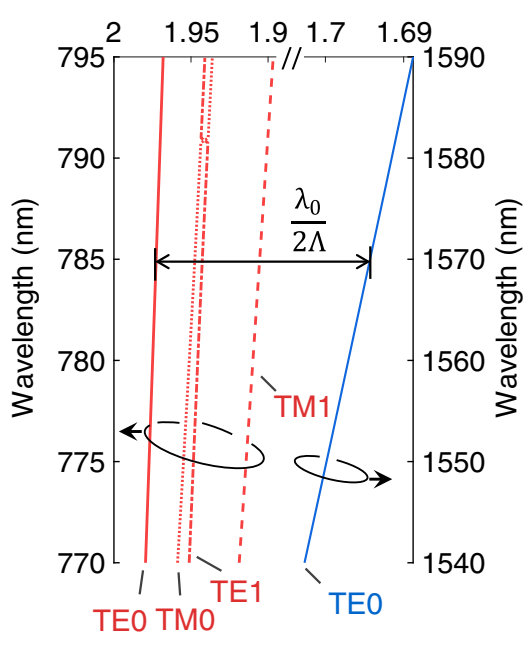

FIG. 1. (a) Optical image of the waveguide with a poled region of length $5 \mathrm{~mm}$ between electrodes. (b) Magnified image of a selected region, also showing the poling electrodes. (c) Cross section of the ridge waveguide. (d) Calculated profile (magnitude of the major electric-field component) of the quasi-TE-polarized fundamental mode at $1570 \mathrm{~nm}$ for the down-converted photons. (e) Calculated profile of the quasi-TE-polarized mode at $785 \mathrm{~nm}$ for the pump. (f) Effective refractive indices with the criterion for type- 0 QPM with period $\Lambda=2.8 \mu \mathrm{m}$ indicated by the horizontal, double-headed arrow. 
negative electrodes, as shown in Fig. 2(a), and the resulting orientation of the domains is indicated by schematic arrows. Such images were acquired using a custom-built confocal scanning second-harmonic (SH) microscopy instrument. The domain walls are shown as dark-colored stripes and in between them are the oppositely oriented domains, which have been poled mainly along $\hat{z}$, with restricted growth along $\hat{y}$ (which is the waveguide axis). Earlier studies have indicated that poled domains in bulk $x$-cut LN may form in a hexagonal cross section [23,24] which will spread with approximately the same speed in all three equivalent $y$ directions, in contrast to vertical domain growth in traditional $z$-cut LN devices. The poling duty cycle is estimated as $55.2 \% \pm 3.0 \%$, based on the $\mathrm{SH}$ images generated from five different positions along the waveguide, each with an area of $100 \mu \mathrm{m}$ (along the $y$ axis) $\times 30 \mu \mathrm{m}$ (along the $z$ axis), part of which is shown in Fig. 2(a). In order to determine whether LN was poled adequately in depth, we performed a quantitative analysis of the line scans of images; one example is indicated using a red line in Fig. 2(a). Physics-based modeling and key differences with regard to traditional SH microscopy of domain walls (see, e.g., Ref. $[25,26]$ ) are discussed in detail in Ref. [27]. On this sample, we inferred that the poling depth was closer to $245 \mathrm{~nm}$ than either 240 or $250 \mathrm{~nm}$, i.e., achieving about $5 \mathrm{~nm}$ depth resolution as shown in Fig. 2(b). Using such nondestructive, high-resolution 3D diagnostic information, we verified the poling quality over the waveguide. In contrast to our technique, traditional depth-resolved poling diagnostic methods such as etching waveguides using acid to reveal the domain orientation [28] are destructive in nature, typically only revealing data of a poled structure that is no longer useful as a working optical device.

The fabricated waveguide was tested for its properties as a source of optically pumped entangled photon pairs and heralded single-photon generation; see the Supplemental Material for more information [29]. The on-chip pair flux or coincidence rate, which is abbreviated in the figure labels as the pair coincidence rate (PCR), was calculated by dividing the time-averaged value of the measured coincidence rate $N_{\text {SI }}$ by the edge coupling efficiency of the chip, the transmission of the filters, and the detector efficiencies. These quantities were measured by separate calibration measurements. We calculated the PCR for several different values of the input pump power, and Fig. 3(a) plots the result and the fitted line whose slope is $45 \mathrm{MHz} \mathrm{mW}^{-1}$, which is higher than that of a typical value for a state-of-the-art traditional PPLN waveguide of $14 \mathrm{MHz} \mathrm{mW}^{-1}$ [56]. Dividing further by the FWHM of the filter, $\Delta \lambda=0.8 \mathrm{~nm}(100 \mathrm{GHz})$, the brightness is $B=5.6 \times 10^{7}$ pairs s $\mathrm{nm}^{-1} \mathrm{~mW}^{-1}$, or $B=4.6 \times 10^{5}$ pairs s${ }^{-1} \mathrm{GHz}^{-1} \mathrm{~mW}^{-1}$, depending on the units used.

Two photon cross-correlation.-Figure 3(b) shows the measured coincidences-to-accidentals ratio (CAR) versus the PCR. The CAR was calculated as $\mathrm{CAR}=$ $\max \left[g_{\mathrm{SI}}^{(2)}(t)\right]-1$ from the normalized signal-idler crosscorrelation $g_{\mathrm{SI}}^{(2)}(t)$, which was obtained from the histogram of signal-idler coincidences measured as a function of the interchannel delay $t$. The highest CAR was $67224 \pm 714$ measured when the PCR was $7.6 \pm 2.6 \times 10^{4}$ pairs s$^{-1}$ (detected pair flux $24 \pm 8$ pairs s$^{-1}$ ). At the highest power values used here, CAR $=668 \pm 1.7$, at $\mathrm{PCR}=$ $11.4 \pm 0.4 \times 10^{6}$ pairs s$^{-1} \quad$ (detected pair flux $3.6 \pm$ $0.13 \times 10^{3}$ pairs s$^{-1}$ ). The CAR decreased at higher pump powers and thus, at higher PCR, as expected, following the trend line $\mathrm{CAR} \propto \mathrm{PCR}^{-1}$, as shown in Fig. 3. These numbers are significantly higher that which has been achieved using other integrated photonics platforms at $1550 \mathrm{~nm}$ wavelengths, including silicon [15] and silicon nitride [57], measured using similar experiments and apparatus. Compared to traditional PPLN waveguides, the highest reported value (to our knowledge) of CAR is $8 \times 10^{5}$ measured, however, at PCR of only 5 pairs s$^{-1}$ [58]; in comparison, the product of CAR and pair flux is about 3 orders of magnitude higher in our TFLN waveguides, highlighting the efficiency of nanoscale LN waveguides with good QPM properties in generating high quality photon pairs.
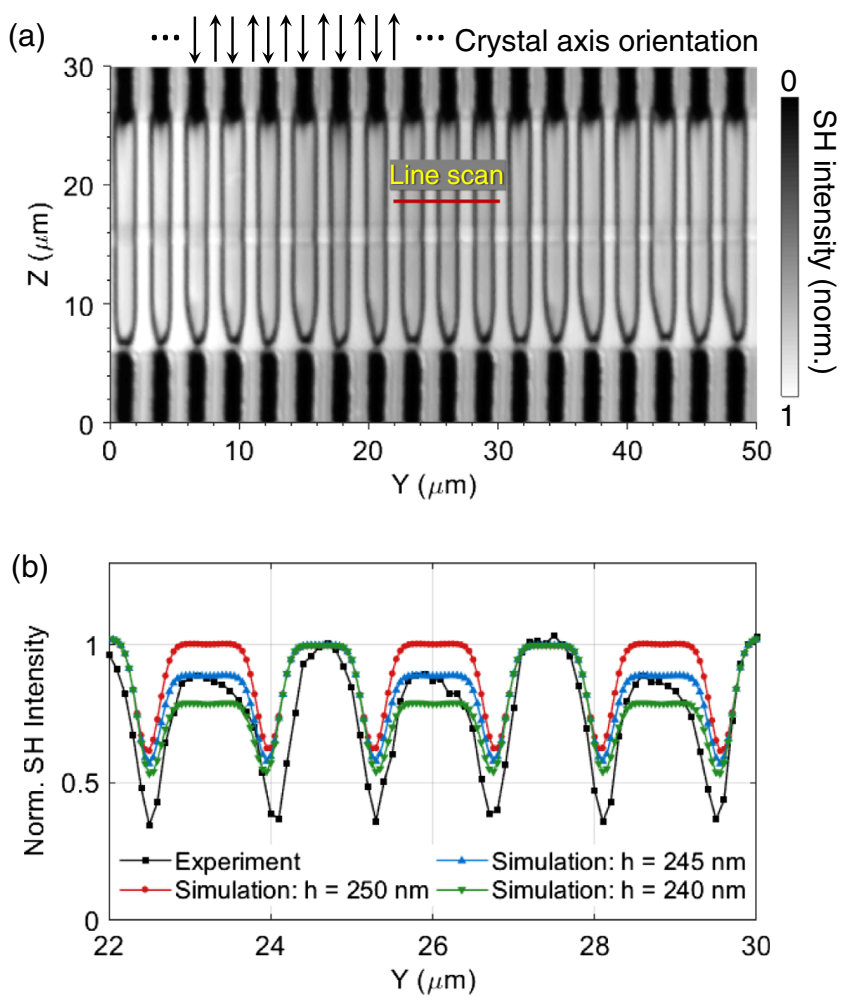

FIG. 2. (a) High-resolution confocal second-harmonic microscopy of the poled domains. The outline of the waveguide can be seen around $Z=16 \mu \mathrm{m}$. The waveguide width $(1.24 \mu \mathrm{m})$ is shorter than the width of the poled domains $(20 \mu \mathrm{m})$. (b) Analysis of a typical optical line scan and best-fit comparison with calculated models (three different assumptions about the poling depth $h$ ). 
(a)
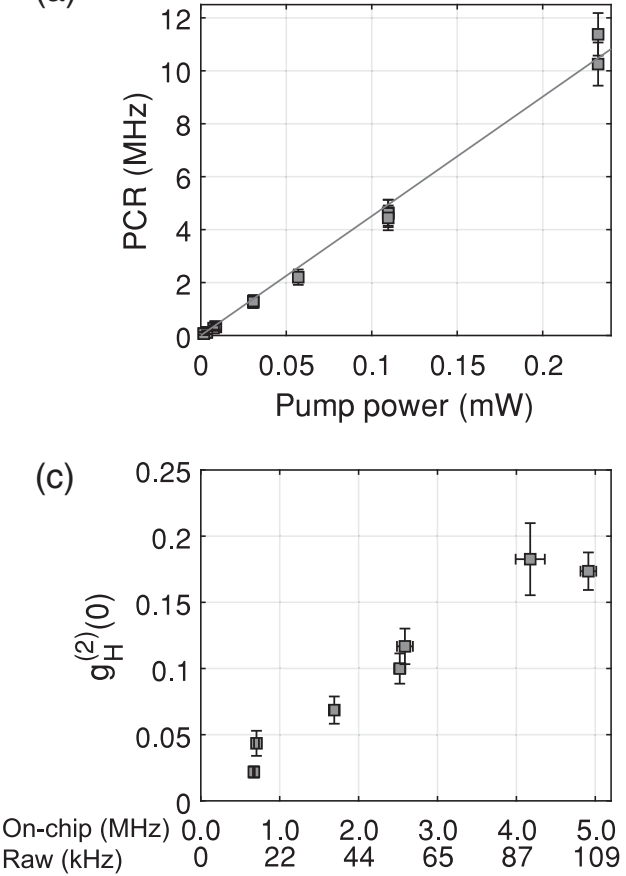

(b)

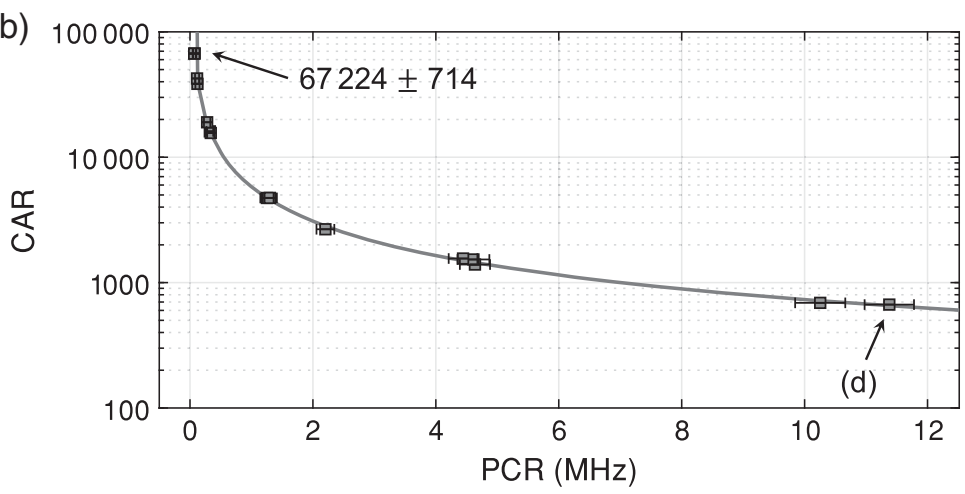

(d)

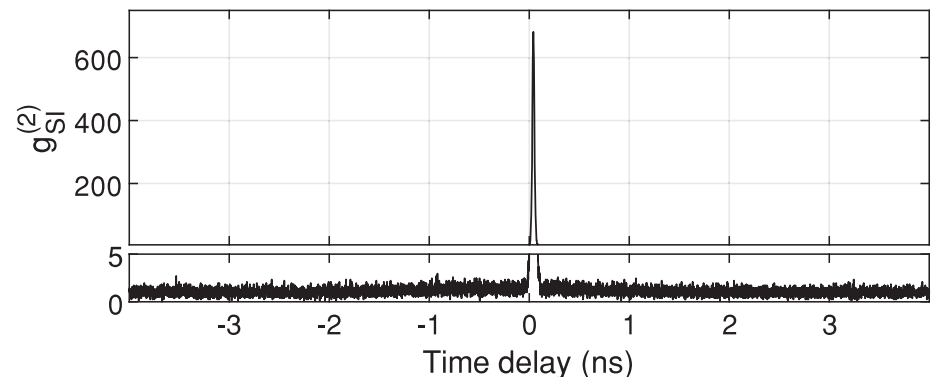

FIG. 3. (a) Pair coincidence rate (PCR, units of MHz) versus pump power (units of $\mathrm{mW}$ ) in the waveguide. (b) Coincidences-toaccidentals ratio (CAR) versus PCR. The highest measured value is indicated. The error bars are one standard deviation in each direction. (c) Heralded single-photon generation. Conditional self-correlation (heralded autocorrelation) $g_{H}^{(2)}(0)$. The horizontal axis shows both the raw herald rate $(\mathrm{kHz})$ and on-chip singles rate $(\mathrm{MHz})$; the latter is obtained by dividing by the measured losses between the chip and the detector. The error bars are one standard deviation. The lowest measured $g_{H}^{(2)}(0)$ was $0.022 \pm 0.004$. (d) The signal-idler cross-correlation histogram for the highest PCR value, indicated by the annotation (d) in (b). The full width at half maximum of the central peak is $27 \mathrm{ps}$.

Heralded single photons.-Detecting one photon of the pair results in projecting the remaining photon into a multimode heralded single-photon state, and this photon is expected to show nonclassical antibunching behavior. Figure 3(c) shows the heralded (i.e., conditional) singlephoton second-order self-correlation function $g_{H}^{(2)}(0)$ obtained by detecting one of the generated photon pair as a herald, and measuring the self-correlation of the other photon in the presence of the herald. Even at the highest power values used in this sequence of measurements, $g_{H}^{(2)}(0)=0.183 \pm 0.03$, well below the classical threshold, at an on-chip (i.e., inferred) heralding rate of $N_{H}=$ $4.2 \mathrm{MHz}$ (raw measured herald rate $91 \mathrm{kHz}$ ). At lower pump powers, values as low as $g_{H}^{(2)}(0)=0.022 \pm 0.004$ were directly measured (the error bar is one standard deviation uncertainty), for an on-chip heralding rate of $N_{H}=0.7 \mathrm{MHz}$ (raw measured herald rate $15 \mathrm{kHz}$ ). There have not been previous reports of heralded single-photon generation using TFLN devices. For comparison with traditional PPLN devices, $g_{H}^{(2)}(0)=0.023$ has been measured at $N_{H}=2.1 \mathrm{MHz}$ [30], and $g_{H}^{(2)}(0)=0.005$ has been measured at (detected) $N_{H}=10 \mathrm{kHz}$ [59].

Entanglement.-The generated photon pair is expected to demonstrate energy-time entanglement which can be investigated through a Franson-type two-photon

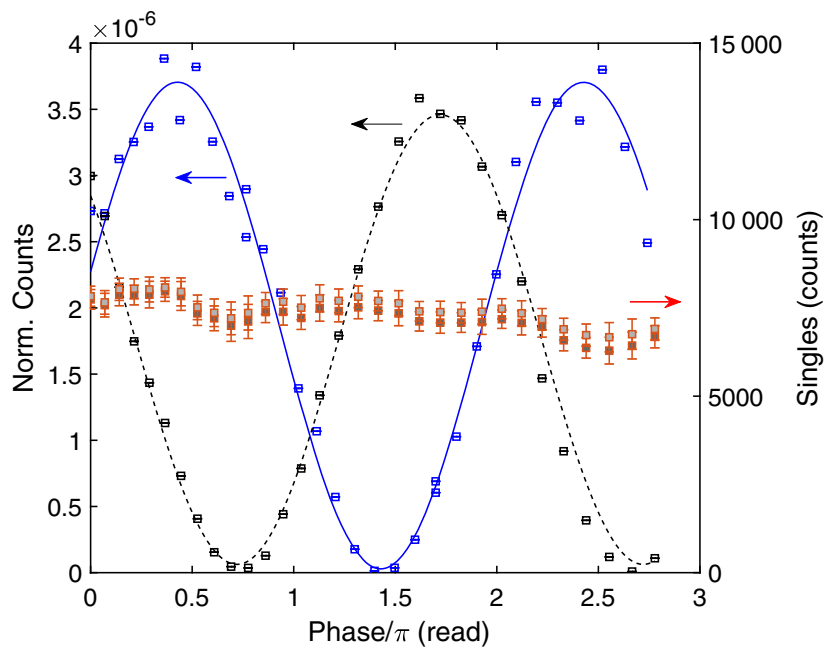

FIG. 4. Two-photon interference pattern measured as the phase of one delay line interferometer (DLI) is swept. The interference pattern for two different phase settings on the second DLI are shown. Black and blue dots (with error bars), experimental data; blue solid and black dashed lines, sinusoidal fit. The right-hand side axis shows the singles counts averaged over the acquisition time, measured at the same time as the two-photon coincidences. 
interference experiment, by violating Bell's inequality [31,32]. Figure 4 shows the measurement of two-photon interference visibility fringes using an unfolded Franson configuration. Proof of photon pair entanglement requires a Clauser-Horne two-photon interference pattern fringe visibility $V \geq 70.7 \%$ (without necessarily providing a test of local realism) [60]. The fitted measurements showed $V$ clearly in excess of this threshold value, measured when the on-chip PCR was about $1 \mathrm{MHz}$, as inferred from the recorded average singles rates and the coupling losses. From a nonlinear least-square curve fit, we obtained $V_{\text {fit }}=$ $98.4 \%$ and $V_{\text {fit }}=96.4 \%$ for the two cases. From the raw data, we calculated $V_{\text {data }}=99.3 \pm 1.9 \%$ (data points shown in blue) and $V_{\text {data }}=99.5 \pm 1.8 \%$ (data points shown in black) for the two phase settings of the unfolded Franson configuration. The indicated error bar is the uncertainty which arises from the goodness of fit of the parameters of the Gaussian function used to fit the central peak; in many cases, the size of the error bar is too small to be visible. These measurements confirmed the high quality energy-time entanglement properties of the pairs, as shown by the sinusoidal variation of coincidences with phase, and in both cases, the flat singles rates show the absence of single-photon interference $[31,32]$.

In conclusion, as shown in Table I, high values of CAR, as well as the first reports of low values of $g_{H}^{(2)}(0)$ and high values of $V$, have been made for entangled photon pairs at telecommunications wavelengths generated using poled nanoscale thin-film LN waveguides. The ability to perform high-resolution in situ imaging and analysis of the poled domain structure helps in identifying the regions of the poled thin-film crystal where the waveguides should be formed. Since this diagnostic technique is nondestructive, it could be useful for the future development of more complex and multicomponent quantum photonic circuits using poled TFLN. We have demonstrated that such waveguides can generate several million photon pairs per second at telecommunications wavelengths with high quality using much less than a milliwatt of pump power. This significant power reduction, by 2 or more orders of magnitude, compared to bulk crystals and traditional SPDC waveguides without sacrificing the high figures of merit (CAR, visibility, etc.) that is expected from traditional LN pair sources, is beneficial for future scale up of on-chip quantum circuits.

This work was supported by National Science Foundation (NSF) (EFMA-1640968, CNS-1525090) and Sandia National Laboratories (Contract Agreement No. 1655264). The partial etch of the LN layer was performed by Q. Lin, U. Javid, J. Ling, and M. Li (University of Rochester).

*smookherjea@ucsd.edu

[1] D. Bouwmeester, J.-W. Pan, K. Mattle, M. Eibl, H. Weinfurter, and A. Zeilinger, Nature (London) 390, 575 (1997).
[2] W. Tittel, J. Brendel, H. Zbinden, and N. Gisin, Phys. Rev. Lett. 81, 3563 (1998).

[3] D. Bouwmeester, J.-W. Pan, M. Daniell, H. Weinfurter, and A. Zeilinger, Phys. Rev. Lett. 82, 1345 (1999).

[4] T. Jennewein, C. Simon, G. Weihs, H. Weinfurter, and A. Zeilinger, Phys. Rev. Lett. 84, 4729 (2000).

[5] P. Walther, K. J. Resch, T. Rudolph, E. Schenck, H. Weinfurter, V. Vedral, M. Aspelmeyer, and A. Zeilinger, Nature (London) 434, 169 (2005).

[6] L. K. Shalm, E. Meyer-Scott, B. G. Christensen, P. Bierhorst, M. A. Wayne, M. J. Stevens, T. Gerrits, S. Glancy, D. R. Hamel, M. S. Allman et al., Phys. Rev. Lett. 115, 250402 (2015).

[7] M. Giustina, M. A. M. Versteegh, S. Wengerowsky, J. Handsteiner, A. Hochrainer, K. Phelan, F. Steinlechner, J. Kofler, J. Å. Larsson, C. Abellán et al., Phys. Rev. Lett. 115, 250401 (2015).

[8] J.-G. Ren, P. Xu, H.-L. Yong, L. Zhang, S.-K. Liao, J. Yin, W.-Y. Liu, W.-Q. Cai, M. Yang, L. Li et al., Nature (London) 549, 70 (2017).

[9] A. L. Migdall, D. Branning, and S. Castelletto, Phys. Rev. A 66, 053805 (2002).

[10] J. L. O’Brien, A. Furusawa, and J. Vučković, Nat. Photonics 3, 687 (2009).

[11] X. S. Ma, S. Zotter, J. Kofler, T. Jennewein, and A. Zeilinger, Phys. Rev. A 83, 043814 (2011).

[12] T. Suhara, Laser Photonics Rev. 3, 370 (2009).

[13] A. Christ, A. Fedrizzi, H. Hübel, T. Jennewein, and C. Silberhorn, in Single-Photon Generation and Detection, edited by A. Migdall, S. Polyakov, J. Fan, and J. Bienfang (Elsevier Inc., Waltham, 2013), Chap. 11, pp. 351-410.

[14] E. Engin, D. Bonneau, C. M. Natarajan, A. S. Clark, M. G. Tanner, R. H. Hadfield, S. N. Dorenbos, V. Zwiller, K. Ohira, N. Suzuki et al., Opt. Express 21, 27826 (2013).

[15] C. Ma, X. Wang, V. Anant, A. D. Beyer, M. D. Shaw, and S. Mookherjea, Opt. Express 25, 32995 (2017).

[16] P. Main, P. J. Mosley, W. Ding, L. Zhang, and A. V. Gorbach, Phys. Rev. A 94, 063844 (2016).

[17] I. W. Frank, J. Moore, J. K. Douglas, R. Camacho, and M. Eichenfield, in Proceedings of the Conference on Lasers and Electro-Optics (CLEO) (Optical Society of America, Washington, 2016), p. SM2E.6.

[18] R. Luo, H. Jiang, S. Rogers, H. Liang, Y. He, and Q. Lin, Opt. Express 25, 24531 (2017).

[19] A. Rao, N. Nader, M. J. Stevens, T. Gerrits, O. S. MagañaLoaiza, G. F. Camacho-González, J. Chiles, A. Honardoost, M. Malinowski, R. Mirin et al., in Proceedings of the Conference on Lasers and Electro-Optics (CLEO) (Optical Society of America, Washington, 2018), p. JTh3C.2.

[20] J.-y. Chen, Y. Meng Sua, Z.-h. Ma, C. Tang, Z. Li, and Y.-p. Huang, OSA Continuum 2, 2914 (2019).

[21] S. B. Elkus, K. Abdelsalam, A. Rao, V. Velev, S. Fathpour, P. Kumar, and S. G. Kanter, Opt. Express 27, 38521 (2019).

[22] L. E. Myers, R. C. Eckardt, M. M. Fejer, R. L. Byer, W. R. Bosenberg, and J. W. Pierce, J. Opt. Soc. Am. B 12, 2102 (1995).

[23] G. Berth, V. Wiedemeier, K. P. Hüsch, L. Gui, H. Hu, W. Sohler, and A. Zrenner, Ferroelectrics 389, 132 (2009).

[24] L. Gui, H. Hu, M. Garcia-Granda, and W. Sohler, Opt. Express 17, 3923 (2009). 
[25] G. Berth, V. Quiring, W. Sohler, and A. Zrenner, Ferroelectrics 352, 78 (2007).

[26] J. Kaneshiro, Y. Uesu, and T. Fukui, J. Opt. Soc. Am. B 27, 888 (2010).

[27] M. Ruesing, J. Zhao, and S. Mookherjea, J. Appl. Phys. 126, 114105 (2019).

[28] K. Nassau, H. J. Levinstein, and G. M. Loiacono, Appl. Phys. Lett. 6, 228 (1965).

[29] See Supplemental Material at http://link.aps.org/ supplemental/10.1103/PhysRevLett.124.163603 for additional details, which includes Refs. [13,25,27,28,30-55].

[30] L. A. Ngah, O. Alibart, L. Labonté, V. D’Auria, and S. Tanzilli, Laser Photonics Rev. 9, L1 (2015).

[31] J. D. Franson, Phys. Rev. Lett. 62, 2205 (1989).

[32] P. G. Kwiat, A. M. Steinberg, and R. Y. Chiao, Phys. Rev. A 47, R2472 (1993).

[33] J. Zhao, M. Rüsing, and S. Mookherjea, Opt. Express 27, 12025 (2019).

[34] R. Luo, Y. He, H. Liang, M. Li, and Q. Lin, Optica 5, 1006 (2018).

[35] S. I. Bozhevolnyi, J. M. Hvam, K. Pedersen, F. Laurell, H. Karlsson, T. Skettrup, and M. Belmonte, Appl. Phys. Lett. 73, 1814 (1998).

[36] S. Kurimura and Y. Uesu, J. Appl. Phys. 81, 369 (1997).

[37] M. Flörsheimer, R. Paschotta, U. Kubitscheck, C. Brillert, D. Hofmann, L. Heuer, G. Schreiber, C. Verbeek, W. Sohler, and H. Fuchs, Appl. Phys. B 67, 593 (1998).

[38] P. Mackwitz, M. Rüsing, G. Berth, A. Widhalm, K. Müller, and A. Zrenner, Appl. Phys. Lett. 108, 152902 (2016).

[39] B. J. Rodriguez, R. J. Nemanich, A. Kingon, A. Gruverman, S. V. Kalinin, K. Terabe, X. Y. Liu, and K. Kitamura, Appl. Phys. Lett. 86, 012906 (2005).

[40] E. Soergel, J. Phys. D 44, 464003 (2011).

[41] S. Wang, V. Pasiskevicius, and F. Laurell, Opt. Mater. 30, 594 (2007).

[42] G. Lindgren, A. Zukauskas, V. Pasiskevicius, F. Laurell, and C. Canalias, Opt. Express 23, 20332 (2015).

[43] M. Missey, S. Russell, V. Dominic, R. Batchko, and K. Schepler, Opt. Express 6, 186 (2000).
[44] S. Grilli, P. Ferraro, M. Paturzo, D. Alfieri, and P. De Natale, Opt. Express 12, 1832 (2004).

[45] A. Rosenfeldt and M. Flörsheimer, Appl. Phys. B 73, 523 (2001).

[46] L. Chang, Y. Li, N. Volet, L. Wang, J. Peters, and J. E. Bowers, Optica 3, 531 (2016).

[47] C. Wang, C. Langrock, A. Marandi, M. Jankowski, M. Zhang, B. Desiatov, M. M. Fejer, and M. Lončar, Optica 5, 1438 (2018).

[48] L. G. Helt, M. Liscidini, and J. E. Sipe, J. Opt. Soc. Am. B 29, 2199 (2012).

[49] S. F. Preble, M. L. Fanto, J. A. Steidle, C. C. Tison, G. A. Howland, Z. Wang, and P. M. Alsing, Phys. Rev. Applied 4, 021001 (2015).

[50] M. Beck, J. Opt. Soc. Am. B 24, 2972 (2007).

[51] P. Grangier, G. Roger, and A. Aspect, Europhys. Lett. 1, 173 (1986).

[52] A. B. Uren, C. Silberhorn, J. L. Ball, K. Banaszek, and I. A. Walmsley, Phys. Rev. A 72, 021802(R) (2005).

[53] S. Krapick, H. Herrmann, V. Quiring, B. Brecht, H. Suche, and C. Silberhorn, New J. Phys. 15, 033010 (2013).

[54] A. M. Brańczyk, T. C. Ralph, W. Helwig, and C. Silberhorn, New J. Phys. 12, 063001 (2010).

[55] C. K. Law, I. A. Walmsley, and J. H. Eberly, Phys. Rev. Lett. 84, 5304 (2000).

[56] H. Jin, F. M. Liu, P. Xu, J. L. Xia, M. L. Zhong, Y. Yuan, J. W. Zhou, Y. X. Gong, W. Wang, and S. N. Zhu, Phys. Rev. Lett. 113, 103601 (2014).

[57] X. Lu, Q. Li, D. A. Westly, G. Moille, A. Singh, V. Anant, and K. Srinivasan, Nat. Phys. 15, 373 (2019).

[58] T. Inagaki, N. Matsuda, O. Tadanaga, M. Asobe, and H. Takesue, Opt. Express 21, 23241 (2013).

[59] G. Brida, I. P. Degiovanni, M. Genovese, F. Piacentini, P. Traina, A. Della Frera, A. Tosi, A. Bahgat Shehata, C. Scarcella, A. Gulinatti et al., Appl. Phys. Lett. 101, 221112 (2012).

[60] J. F. Clauser and M. A. Horne, Phys. Rev. D 10, 526 (1974). 